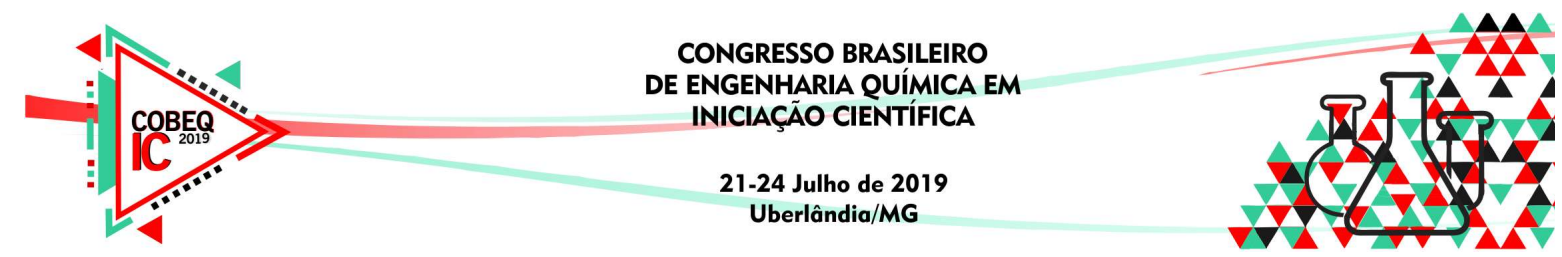

\title{
PREDIÇÃO E COMPUTAÇÃO DAS PROPRIEDADES TERMODINÂMICAS E DE TRANSPORTE DE FLUIDOS PUROS UTILIZANDO EQUAÇÕES CÚBICAS DE ESTADO
}

\author{
R. C. ACOSTA ${ }^{1}$, L. F. SANTOS ${ }^{2}$ e P. ARCE ${ }^{3}$ \\ 1,2,3 Universidade de São Paulo, Escola de Engenharia de Lorena \\ E-mail para contato: rodolfo.ca@alunos.eel.usp.br
}

\begin{abstract}
RESUMO - Neste projeto foi desenvolvido um modelo numérico, utilizando o software Matlab ${ }^{\circledR}$, para predizer propriedades termodinâmicas, através da utilização da equação de estado de Peng-Robinson, que possui comprovada eficiência na modelagem e simulação de processos industriais, e da equação de Span e Wagner. O desenvolvimento do programa é importante pois sabe-se que os modelos termodinâmicos baseados em equações cúbicas de estado são as melhores ferramentas para a predição de propriedades termodinâmicas e de transporte para fluidos puros.
\end{abstract}

\section{INTRODUÇÃO}

A escolha de um trabalho baseado em equações cúbicas de estado foi devido ao fato de que essas equações são as melhores ferramentas para a predição de propriedades termodinâmicas e de transporte.

As propriedades termodinâmicas têm fundamental importância para a realização de cálculos de balanço de massa, energia e momentum, além de serem necessárias para predizer o escoamento de fluidos, sendo amplamente utilizadas por cientistas e engenheiros.

O desenvolvimento de um programa computacional tem por finalidade tornar a obtenção de propriedades termodinâmicas mais prática, além do desenvolvimento de uma interface gráfica para facilitar a compreensão pelo usuário.

\subsection{Equações de estado}

Uma equação cúbica de estado é o polinômio mais simples capaz de fornecer o limite do gás ideal a volume infinito e de representar os volumes molares das fases líquida e vapor em forma simultânea. As equações cúbicas de estado possuem dois termos característicos: "a" e "b". O primeiro parâmetro se identifica com as forças intermoleculares entre as moléculas (forças repulsivas) e o segundo leva em consideração as forças de atração entre as moléculas. Ambos os parâmetros podem ser determinados através das seguintes relações no ponto crítico, expressas pela Equação 1 (Michelsen e Mollerup, 2007):

$$
\left(\frac{\partial P}{\partial V}\right)_{T_{c}}=\left(\frac{\partial^{2} P}{\partial V^{2}}\right)_{T_{c}}=0
$$




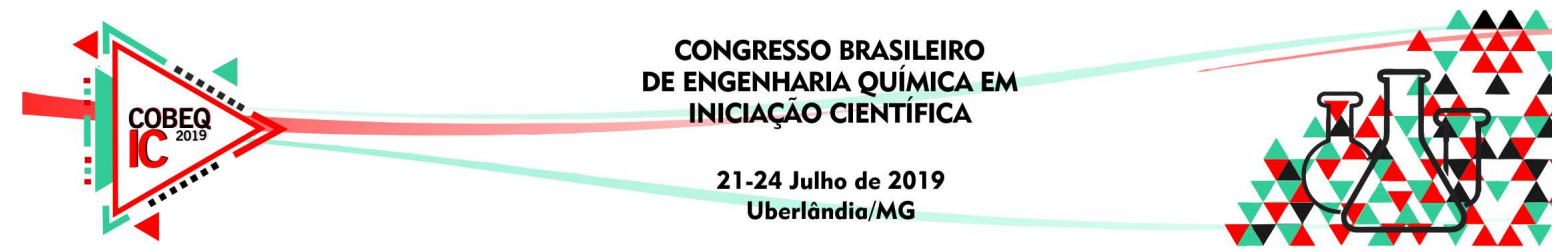

É importante também salientar que as equações cúbicas de estado, principalmente a equação de Peng-Robinson, não apenas são capazes de modelar as fases líquida e vapor, mas também a fase sólida em equilíbrio com as fases líquida, vapor ou gás (Arce, 2005).

A equação de Peng-Robinson e sua forma polinomial são expressas pelas equações 2 e 3 .

$$
\mathrm{P}=\frac{\mathrm{RT}}{\mathrm{v}-\mathrm{b}}-\frac{\mathrm{a}_{\mathrm{c}} \alpha(\mathrm{T})}{\mathrm{v}(\mathrm{v}+\mathrm{b})+\mathrm{b}(\mathrm{v}-\mathrm{b})}
$$

Onde $\alpha=\left(1+\left(0.37464+1.54226 \omega-0.26992 \omega^{2}\right)(1-\operatorname{Tr} 0.5)\right)^{2}, \mathrm{a}=0.45724 \mathrm{R}^{2} \mathrm{Tc}^{2} / \mathrm{Pc}^{2} \mathrm{e}$ $\mathrm{b}=0.0778 \mathrm{RTc} / \mathrm{Pc}$.

$$
\mathrm{Z}^{3}-(1-\mathrm{B}) \mathrm{Z}^{2}+\left(\mathrm{A}-2 \mathrm{~B}-3 \mathrm{~B}^{2}\right) \mathrm{Z}-\mathrm{AB}+\mathrm{B}^{2}+\mathrm{B}^{3}=0
$$

Onde $\mathrm{A}=\mathrm{a} \alpha \mathrm{P} / \mathrm{R}^{2} \mathrm{~T}^{2}$ e $\mathrm{B}=\mathrm{bP} / \mathrm{TR}$.

\subsection{Energia de Helmholtz}

A energia livre de Helmholtz é uma grandeza que mensura a quantidade de energia interna disponível em um sistema que pode ser utilizada na forma de trabalho. O conceito teve origem na verificação de que nem toda energia interna de um sistema é passível de produzir trabalho, pois uma parcela desta energia está associada à entropia do sistema (Callen, 1985).

A equação de energia de Helmholtz A, pode ser expressa com duas variáveis independentes, densidade $\rho$ e temperatura T. A energia adimensional de Helmholtz $\Phi=\mathrm{A} /(\mathrm{RT})$ é comumente dividida em uma parte que depende do comportamento do gás ideal e uma parte que leva em conta o comportamento do fluido, como se pode observar pela equação 4:

$$
\Phi(\delta, \tau)=\Phi 0(\delta, \tau)+\Phi r(\delta, \tau)
$$

Onde $\delta=\rho / \rho c$ é a densidade reduzida e $\tau=\mathrm{Tc} / \mathrm{T}$ é a inversa da temperatura reduzida. Ambas a densidade e a temperatura são reduzidas com seus valores críticos, $\rho c$ e Tc respectivamente (Span e Wagner, 1996).

\section{METODOLOGIA}

\subsection{Matlab ${ }^{\circledR}$}

Para o desenvolvimento do programa, foi utilizado o software Matlab ${ }^{\circledR}$ desenvolvido pela empresa norte americana MathWorks, fundada em 1984 e responsável por desenvolver softwares matemáticos para engenheiros e cientistas (Moler, 2004).

\subsection{Funções}

Foram desenvolvidas quatro funções para ajudar no funcionamento do software, "PengRobinson", "FiIdeal", "FiReal" e "Psat". 


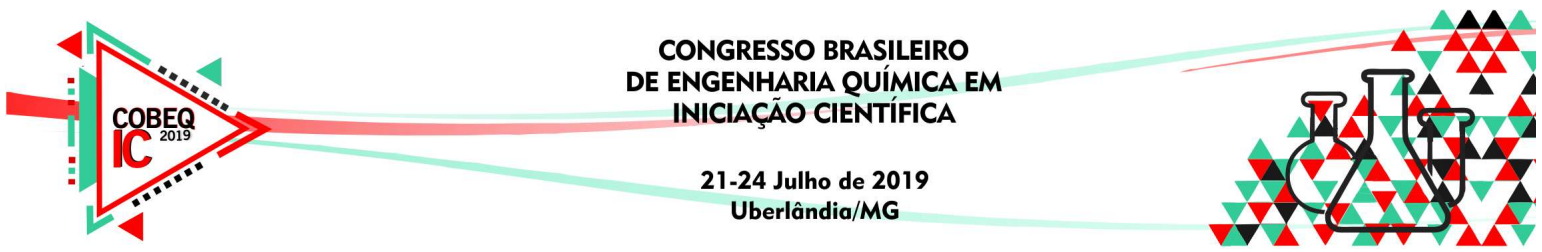

A primeira função, "PengRobinson", representa a o cálculo da equação de Peng-Robinson vista nas equações 2 e 3 . A função recebe os valores de temperatura $T$ e pressão $P$ do sistema, a temperatura crítica Tc e a pressão crítica $\mathrm{Pc}$, e o fator acêntrico $\mathrm{w}$ do fluido e retorna o fator de compressibilidade $\mathrm{Z}$.

A função "FiIdeal" representa o cálculo ideal da energia de Helmholtz e recebe os valores da densidade reduzida $\delta$ e da inversa da temperatura reduzida $\tau$, e retorna o valor numérico de $\Phi^{0}$ e suas derivadas.

Já a função "FiReal" representa a parte real da energia de Helmholtz. Semelhante a função "FiIdeal", ela também recebe os valores da densidade reduzida $\delta$ e da inversa da temperatura reduzida $\tau$, e retorna o valor numérico de $\Phi^{\mathrm{r}}$ e suas derivadas.

A última função "Psat" foi criada a partir da equação de Peng Robinson e recebe os valores de temperatura $\mathrm{T}$ do sistema, a temperatura crítica Tc e a pressão crítica Pc, e o fator acêntrico $\omega$ do fluido e retorna uma estimativa do valor da pressão saturada Psat na temperatura indicada.

\subsection{Interface Gráfica}

A interface do software foi criada com o auxílio do aplicativo GUIDE (Graphical User Interface Development Environment), que significa ambiente de desenvolvimento de interface gráfica de usuário. O Layout do programa pode ser visto de acordo com a Figura 1.

Figura 1 - Layout da Interface Gráfica.

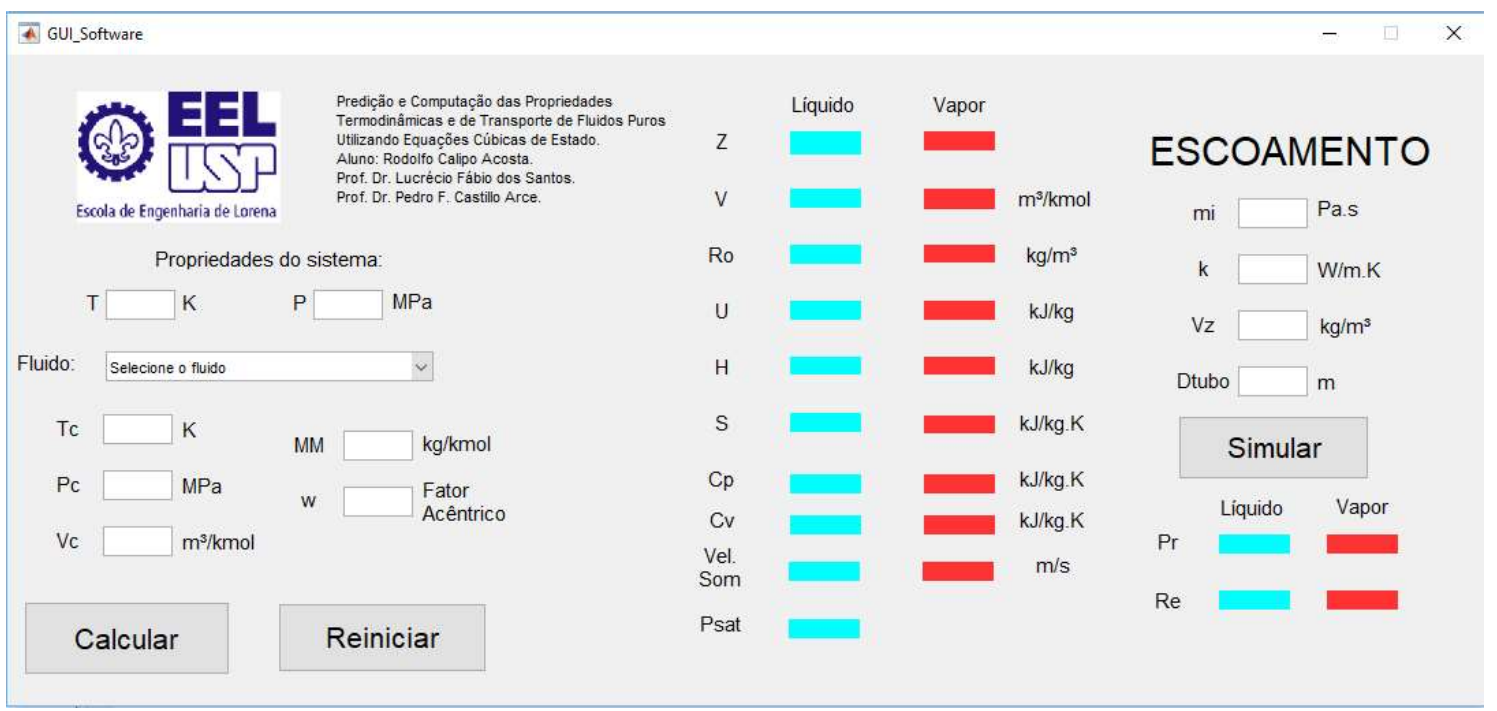

Para o funcionamento do programa, cada caixa em branco recebe e armazena um valor, também há um botão pop up em que é possível escolher um fluido e suas propriedades são plotadas automaticamente na tela. A programação do botão pop up foi feita armazenando dados fornecidos por Yaws (1999) em forma de vetor. 


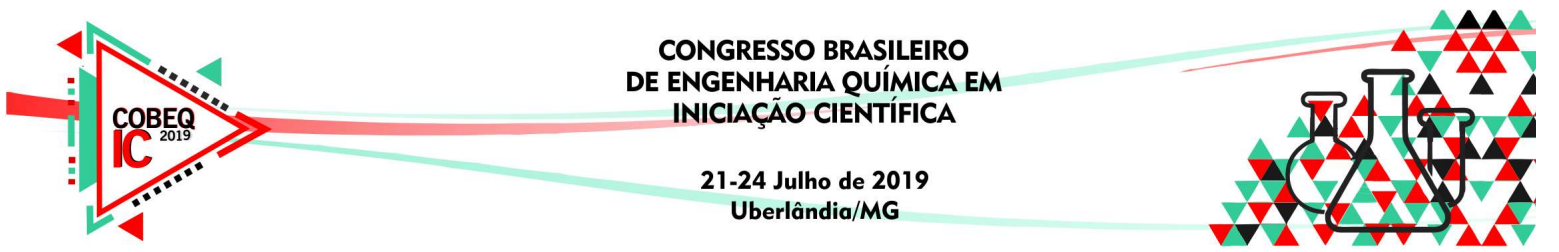

No botão "Calcular" é onde se encontra a grande parte dos cálculos realizadas pelo programa. Primeiro ele recebe os valores digitados na interface, e se a condição do sistema for inferior as condições críticas, o programa estima a pressão de saturação para a temperatura informada. Logo após, ele realiza o cálculo do fator de compressibilidade $\mathrm{Z}$ utilizando a função "PengRobinson", depois são calculados os valores de $\Phi^{0}$ e $\Phi^{\mathrm{r}}$ e as propriedades do fluido que retornam nas caixas em azul e vermelho.

\section{ANÁLISE E DISCUSSÃO DOS RESULTADOS}

Para análise dos resultados obtidos pelo programa, escolheu-se o dióxido de carbono como substância principal, pois a equação da parte residual da energia de Helmholtz foi baseada nessa substância. Os dados foram comparados aos fornecidos por Span e Wagner (1996).

Para realizar o teste, escolheu-se pressões e temperaturas aleatórias, porém baseando-se no ponto crítico.

A primeira pressão analisada, foi a de $0,1 \mathrm{MPa}$, pois está bem abaixo da pressão crítica $(7,38 \mathrm{MPa})$, as temperaturas escolhidas foram $200 \mathrm{~K}$ por ser uma temperatura mais baixa e acima do ponto de fusão $(194,525 \mathrm{~K}), 300 \mathrm{~K}$ por ser próxima do ponto crítico $(304,19 \mathrm{~K})$ e $700 \mathrm{~K}$ por ser bem acima do ponto crítico.

Com os dados obtidos pelo programa e pela literatura, foi montada a Tabela 1 para se realizar a comparação dos valores e indicar o erro relativo.

Tabela 1 - Comparação para os dados obtidos pelo programa com $\mathrm{P}=0,1 \mathrm{MPa}$

\begin{tabular}{|c|c|c|c|c|c|c|c|c|c|}
\hline \multirow[t]{2}{*}{$\mathrm{P}=0,1 \mathrm{Mpa}$} & \multicolumn{3}{|c|}{$T=200 \mathrm{~K}$} & \multicolumn{3}{|c|}{$\mathrm{T}=300 \mathrm{~K}$} & \multicolumn{3}{|c|}{$\mathrm{T}=700 \mathrm{~K}$} \\
\hline & Teórico & Programa & Erro & Teórico & Programa & Erro & Teórico & Programa & Erro \\
\hline Ro $\left(\mathrm{kg} / \mathrm{m}^{3}\right)$ & 2,698 & 2,6925 & $0,20 \%$ & 1,773 & 1,7739 & $0,05 \%$ & 0,75619 & 0,7561 & $0,01 \%$ \\
\hline$U(\mathbf{k} \mathbf{J} / \mathbf{k g})$ & $-117,11$ & $-117,15$ & $0,03 \%$ & $-55,751$ & $-55,668$ & $0,15 \%$ & 271,13 & 271,05 & $0,03 \%$ \\
\hline $\mathrm{H}(\mathbf{k J} / \mathbf{k g})$ & $-80,049$ & $-80,087$ & $0,05 \%$ & 0,64941 & 0,7348 & $13,15 \%$ & 403,38 & 403,29 & $0,02 \%$ \\
\hline S (kJ/kg.K) & $-0,32046$ & $-0,3199$ & $0,17 \%$ & 0,00559 & 0,006 & $7,33 \%$ & 0,84226 & 0,8423 & $0,00 \%$ \\
\hline Cv (kJ/kg.K) & 0,56339 & 0,5633 & $0,02 \%$ & 0,65932 & 0,614 & $6,87 \%$ & 0,93752 & 0,9374 & $0,01 \%$ \\
\hline Cp (kJ/kg.K) & 0,77091 & 0,7707 & $0,03 \%$ & 0,85253 & 0,8054 & $5,53 \%$ & 1,1269 & 1,1268 & $0,01 \%$ \\
\hline Vel Som $(\mathrm{m} / \mathrm{s})$ & 223 & 223,01 & $0,00 \%$ & 269,39 & 271,34 & $0,72 \%$ & 398,68 & 398,68 & $0,00 \%$ \\
\hline Psat (MPa) & - & - & - & 6,7131 & 6,7162 & $0,05 \%$ & - & - & - \\
\hline
\end{tabular}

Pode-se notar que para a temperatura de $200 \mathrm{~K}$ o erro relativo foi baixo em todas as propriedades termodinâmicas, com erros inferiores a $0,2 \%$ e extremamente baixo para $700 \mathrm{~K}$, com erros inferiores a $0,03 \%$. Porém, na temperatura de $300 \mathrm{~K}$ foi encontrado erros razoáveis, com valor máximo de $13,15 \%$ para a energia interna.

Os erros maiores para a temperatura de $300 \mathrm{~K}$ se deve ao fato de que as propriedades termodinâmicas trabalhadas nesse projeto estão em função da densidade reduzida $\rho$ e do inverso da temperatura reduzida $\tau$, portanto, na região próxima ao ponto crítico, pequenas variações nessas propriedades causam grandes diferenças nas propriedades termodinâmicas, dificultando a precisão do programa. 


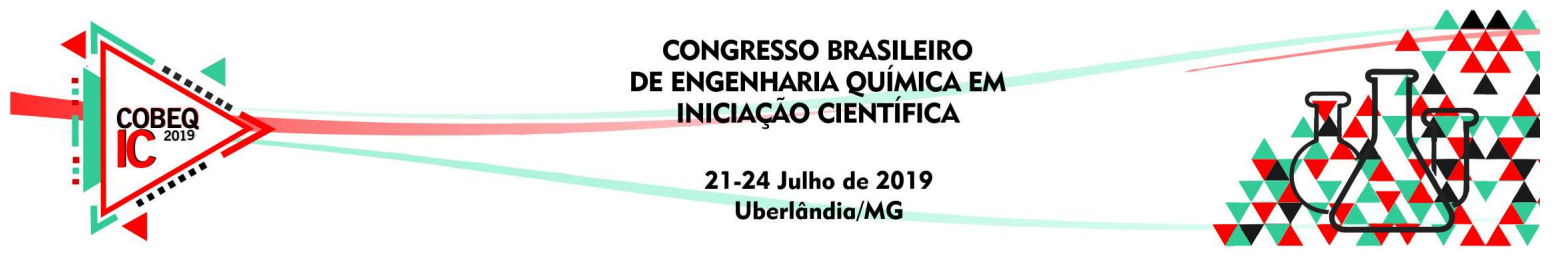

A próxima pressão escolhida foi a de $5 \mathrm{MPa}$, sendo um pouco próxima da temperatura crítica. Para pressões maiores, não é viável utilizar temperaturas abaixo de $300 \mathrm{~K}$, pois o ponto de ebulição aumenta com o aumento da pressão, limitando as opções de temperaturas abaixo do ponto crítico. As temperaturas utilizadas foram de $300 \mathrm{~K}, 500 \mathrm{~K}$ e $700 \mathrm{~K}$

Com os dados obtidos pelo programa e pela literatura, foi montada a Tabela 2 para se realizar a comparação dos valores e indicar o erro relativo.

Tabela 2 - Comparação para os dados obtidos pelo programa com $\mathrm{P}=5 \mathrm{MPa}$

\begin{tabular}{|c|c|c|c|c|c|c|c|c|c|}
\hline \multirow[t]{2}{*}{$\mathrm{P}=5 \mathrm{Mpa}$} & \multicolumn{3}{|c|}{$T=300 \mathrm{~K}$} & \multicolumn{3}{|c|}{$\mathrm{T}=500 \mathrm{~K}$} & \multicolumn{3}{|c|}{$\mathrm{T}=700 \mathrm{~K}$} \\
\hline & Teórico & Programa & Erro & Teórico & Programa & Erro & Teórico & Programa & Erro \\
\hline $\operatorname{Ro}\left(\mathrm{kg} / \mathrm{m}^{3}\right)$ & 128,4 & 131,54 & $2,45 \%$ & 54,826 & 54,96 & $0,24 \%$ & 37,823 & 37,766 & $0,15 \%$ \\
\hline $\mathrm{U}(\mathbf{k J} / \mathrm{kg})$ & $-99,772$ & 370,94 & $471,79 \%$ & 81,644 & 81,559 & $0,10 \%$ & 263,87 & 263,81 & $0,02 \%$ \\
\hline $\mathrm{H}(\mathrm{kJ} / \mathrm{kg})$ & $-60,83$ & 415,7 & $783,38 \%$ & 172,84 & 172,75 & $0,05 \%$ & 396,07 & 396 & $0,02 \%$ \\
\hline S (kJ/kg.K) & $-0,88811$ & 0,6633 & $174,69 \%$ & $-0,28196$ & $-0,2823$ & $0,12 \%$ & 0,09293 & 0,0932 & $0,29 \%$ \\
\hline Cv (kJ/kg.K) & 0,85258 & $-158,27$ & $18663,65 \%$ & 0,84035 & 0,8403 & $0,01 \%$ & 0,94202 & 0,9419 & $0,01 \%$ \\
\hline Cp (kJ/kg.K) & 1,8025 & $-125,73$ & $7075,31 \%$ & 1,084 & 1,084 & $0,00 \%$ & 1,1523 & 1,1521 & $0,02 \%$ \\
\hline Vel Som (m/s) & 223,25 & 163,28 & $26,86 \%$ & 337,43 & 337,42 & $0,00 \%$ & 402,3 & 402,28 & $0,00 \%$ \\
\hline Psat (MPa) & 6,7131 & 6,7162 & $0,05 \%$ & - & - & - & - & - & - \\
\hline
\end{tabular}

Pode-se notar que para as temperaturas de $500 \mathrm{~K}$ e $700 \mathrm{~K}$ os erros relativos foram pequenos, sendo inferiores a $0,3 \%$. Contudo, para o valor de $300 \mathrm{~K}$, as propriedades termodinâmicas apresentaram valores extremamente destoantes do esperado, com erros relativos absurdos chegando a ser superior a $18000 \%$. Mostrando que quanto mais próximo as temperaturas e pressões estiverem do ponto crítico, menos preciso será o programa.

Por fim, tem-se a pressão de $20 \mathrm{MPa}$, bem acima da pressão crítica. Também foram utilizados os valores de $300 \mathrm{~K}, 500 \mathrm{~K}$ e $700 \mathrm{~K}$.

Com os dados obtidos pelo programa e pela literatura, foi montada a Tabela 3 para se realizar a comparação dos valores e indicar o erro relativo.

Tabela 3 - Comparação para os dados obtidos pelo programa com $\mathrm{P}=20 \mathrm{MPa}$

\begin{tabular}{|c|c|c|c|c|c|c|c|c|c|}
\hline \multirow[t]{2}{*}{$\mathrm{P}=20 \mathrm{Mpa}$} & \multicolumn{3}{|c|}{$\mathrm{T}=300 \mathrm{~K}$} & \multicolumn{3}{|c|}{$T=500 \mathrm{~K}$} & \multicolumn{3}{|c|}{$\mathrm{T}=700 \mathrm{~K}$} \\
\hline & Teórico & Programa & Erro & Teórico & Programa & Erro & Teórico & Programa & Erro \\
\hline $\operatorname{Ro}\left(\mathrm{kg} / \mathrm{m}^{3}\right)$ & 905,57 & 911,84 & $0,69 \%$ & 235,24 & 232,9 & $0,99 \%$ & 149,27 & 148,19 & $0,72 \%$ \\
\hline $\mathrm{U}(\mathbf{k J} / \mathbf{k g})$ & $-280,61$ & 33040 & $11874,35 \%$ & 42,292 & 42,763 & $1,11 \%$ & 242,66 & 242,8 & $0,06 \%$ \\
\hline$H(\mathbf{k J} / \mathbf{k g})$ & $-258,52$ & 34476 & $13435,91 \%$ & 127,31 & 127,81 & $0,39 \%$ & 376,64 & 376,74 & $0,03 \%$ \\
\hline$S(\mathbf{k J} / \mathbf{k g} \cdot \mathrm{K})$ & $-1,6334$ & 108,4 & $6736,46 \%$ & $-0,61783$ & $-0,6149$ & $0,47 \%$ & $-0,19756$ & $-0,1957$ & $0,94 \%$ \\
\hline Cv (kJ/kg.K) & 0,91763 & -11247 & $1225757,40 \%$ & 0,88074 & 0,8802 & $0,06 \%$ & 0,95465 & 0,9544 & $0,03 \%$ \\
\hline Cp (kJ/kg.K) & 2,1267 & 2397,2 & $112619,24 \%$ & 1,3228 & 1,3197 & $0,23 \%$ & 1,2253 & 1,2244 & $0,07 \%$ \\
\hline Vel Som (m/s) & 586,17 & 0 & $100,00 \%$ & 350,29 & 349,78 & $0,15 \%$ & 421,46 & 421,15 & $0,07 \%$ \\
\hline Psat (MPa) & 6,7131 & 6,7162 & $0,05 \%$ & - & - & - & - & - & - \\
\hline
\end{tabular}

Notam-se que as temperaturas de $500 \mathrm{~K}$ e $700 \mathrm{~K}$ continuam com erros baixos, próximos a $1 \%$, porém os erros obtidos para $300 \mathrm{~K}$ aumentaram em relação a mesma temperatura para a pressão de $5 \mathrm{MPa}$, sendo o menor erro de $6000 \%$ para a entropia e o maior superior a $1.000 .000 \%$ para o calor específico a volume constante. Isso mostra que se uma das 


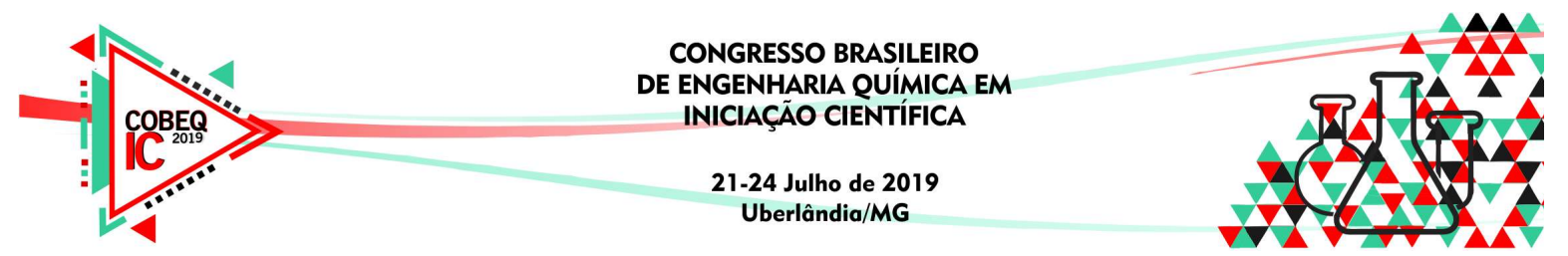

propriedades estiver próxima do ponto crítico e a outra estiver muito distante, a precisão do programa também é prejudicada.

\section{CONCLUSÃO}

De modo geral, o programa funcionou de forma correta e coerente com os dados da literatura, apresentando erros inferiores a $1 \%$ na maior parte do tempo, de acordo com as Tabelas 1, 2 e 3 . Entretanto, na região próxima ao ponto crítico foram encontrados alguns desvios relativos extremamente elevados para alguns dados termodinâmicos, mostrando que nessas regiões, pequenas diferenças nas propriedades reduzidas causam grandes variações nos resultados.

Outro fator que pode ter influenciado nos resultados é a diferença nos dados referentes ao ponto crítico e o fator acêntrico, que apesar de baixos e na escala das casas decimais, acabam resultando em divergências no valor obtido pelo programa e a literatura.

Portanto, pode-se concluir que o programa é capaz de cumprir sua função com precisão, apenas com ressalvas para áreas próximas ao ponto crítico da substancia.

\section{REFERÊNCIAS}

PENG, D.Y.; ROBINSON, D.B. A new two-constant equation of state. Ind. Eng. Chem. Fundam, p. 59-64, 1976.

MICHELSEN, M.L.; MOLLERUP, J.M. Thermodynamic Models: Fundamentals \& Computational Aspects. Tie Line Publications, 2da edição, Dinamarca, 2007.

ARCE, P. Modelagem e Computação do Equilíbrio Multifásico de Fluidos e Fenômenos Críticos em Solubilidades de Polímeros em Misturas de Dióxido de Carbono Supercrítico + Cosolvente. Tese de Doutorado, UNICAMP, Campinas, Brasil, 2005.

CALLEN, H. B. Thermodynamics and an introduction to thermostatics. John Wiley \& Sons. New York USA, 1985.

SPAN, R.; WAGNER, W. A new equation of state for carbon dioxide covering the fluid region from the triple-point temperature to $1100 \mathrm{~K}$ at pressures up to $800 \mathrm{MPa}$. Journal of physical and chemical reference data, v. 25, n. 6, p. 1509-1596, 1996.

MOLER, C. The Origins of MATLAB, MathWorks. (2004).

YAWS C. L. Chemical Properties Handbook. Ed. McGraw Hill, New York, 1999. DIPPR Information and Data Evaluation Manager. Version 1.2.0, 2000. 